\title{
Polynomial Algorithms for Parametric Minquantile and Maxcovering Planar Location Problems with Locational Constraints
}

\author{
Emilio Carrizosa \\ Departamento de Estadística e I.O. \\ Universidad de Sevilla \\ Tarfia s/n, 41012 Sevilla, Spain \\ ecarriz@cica.es \\ Frank Plastria \\ Center for Industrial Location \\ Vrije Universiteit Brussel \\ Pleinlaan, 2, 1050 Brussels, Belgium \\ Frank.Plastria@vub.ac.be
}

\begin{abstract}
A location is sought within some convex region of the plane for the central site of some public service to a finite number of demand points. The parametric maxcovering problem consists in finding for each $R>0$ the point from which the total weight of the demand points within distance $R$ is maximal. The parametric minimal quantile problem asks for each percentage $\alpha$ the point minimising the distance necessary for covering demand points of total weight at least $\alpha$. We investigate the properties of these two closely related problems and derive polynomial algorithms to solve them both in case of either (possibly inflated) Euclidean or polyhedral distances.
\end{abstract}

Key Words: maximal covering, minimal quantile, single facility location, Euclidean distance, polyhedral distance, sensitivity analysis

AMS subject classification: $90 \mathrm{~B} 85,90 \mathrm{C} 31$.

\section{Introduction}

Let $A=\left\{a_{1}, \ldots, a_{p}\right\} \subset \mathbf{R}^{2}$ be a set of demand points, requiring for a certain public service, the location of which is to be determined within a closed convex set $X$. Due to the public nature of the service, the minimisation of the maximum distance seems to be a plausible criterion. However, if some

The research of the first author is partially supported by Grant PB96-1416-C02-02 of Ministerio de Educación y Cultura, Spain

Received: December 1997; Accepted: November 1998 
of the demand points are far away from the rest (outliers), the classical minmax solution might be very unsatisfactory, since large numbers of demand points might suffer from low-level (far off) service only due to full concern for the (few) outlying ones. Very often this leads to an unacceptable low service level for most.

Two ways to avoid this drawback could be :

1. Provide a service as good as possible to a given minimal portion $\alpha \in] 0,1]$ of the potential demand for the service, in detriment of the rest.

2. Fix a standard $R$ (seen as the highest acceptable distance between the server and the demand), and maximize the part of demand within this radius.

We call model 1 . the minimal quantile problem. In spite of its immediate practical interest this type of model seems to be new in the literature on location. Model 2. corresponds to the well-known maximal covering problem, for which some very efficient algorithms have been proposed in the unconstrained case $\left(X=\mathbb{R}^{2}\right)$ and Euclidean distance, see Drezner (1981) and Mehrez-Stulman (1982). Both models, however, rely on the choice of a parameter, either $\alpha$ or $R$. And although having a clear interpretation it may often be felt as artificial to fix such a parameter a priori, without any knowledge of its impact on the quality of the resulting optimal solutions. It follows that it is of high practical interest to obtain a sensitivity analysis with respect to this parameter. The existing solution methodology mentioned above for the maxcovering problem does not readily allow to analyse the effects of modifying the radius $R$.

In this paper we show how both these problems may be solved simultaneously for all $\alpha$ and $R$. After stating the problems formally in section 2, a general algorithm is described in section 3. This algorithm calls for repeated solution of certain subproblems. These subproblems are studied in detail in section 4. The next two sections discuss how the proposed methodology yields polynomial algorithms in the Euclidean distance case and in the polyhedral gauges case respectively. In the concluding section some extended complexity results are stated, and the extension to the location of several facilities is discussed. 


\section{Problem formulations}

For $i \in\{1, \ldots, p\}$, let $\gamma_{i}$ be a gauge in $\mathbb{R}^{2}$ (the asymmetric equivalent to norms, see Durier and Michelot (1985)), and define the function giving the distance of a point $x$ to demand point $a_{i}$ as

$$
f_{i}: \mathbb{R}^{2} \rightarrow \mathbb{R}: x \mapsto f_{i}(x):=\gamma_{i}\left(x-a_{i}\right)
$$

Let $w_{i}>0$ be a weight associated with demand point $a_{i}$, representing e.g. a population, frequency of demand, risk of emergency, etc. Without loss of generality we assume that $\sum_{i=1}^{p} w_{i}=1$.

Consider the functions

$$
C:\left[0,+\infty\left[\times \mathbb{R}^{2} \rightarrow \mathbb{R}_{+}:(R, x) \mapsto C(R, x):=\sum_{i \mid f_{i}(x) \leq R} w_{i}\right.\right.
$$

and

$$
Q:] 0,1] \times \mathbf{R}^{2} \rightarrow \mathbf{R}_{+}:(\alpha, x) \mapsto Q(\alpha, x):=\min _{I \in S_{\alpha}} \max _{i \in I} f_{i}(x)
$$

with $S_{\alpha}=\left\{I \subseteq\{1, \ldots, p\} \mid \sum_{i \in I} w_{i} \geq \alpha\right\} . C(R, x)$ represents the covering, i.e. the total weight of demand points within distance $R$ of $x$, as measured by the $f_{i} . Q(\alpha, x)$ is the smallest distance from $x$ within which at least total weight $\alpha$ of demand points may be found, in other words, the $\alpha$-quantile of the distribution of demand distances from $x$ with the weights as frequencies.

Given $R \in[0,+\infty[$ and $\alpha \in] 0,1]$, let $M C_{R}$ and $M Q_{\alpha}$ be the $R$ maxcovering problem and $\alpha$-minquantile problem defined by :

$$
\begin{aligned}
& \left(M C_{R}\right) \quad C^{*}(R):=\max _{x \in X} C(R, x) \\
& \left(M Q_{\alpha}\right) \quad Q^{*}(\alpha):=\min _{x \in X} Q(\alpha, x)
\end{aligned}
$$

We will call any $x \in X$ such that $C(R, x)=C^{*}(R)\left(\operatorname{resp} . Q(\alpha, x)=Q^{*}(\alpha)\right)$ an $R$-maxcovering point (resp. an $\alpha$-minquantile point). Solving $M C_{R}$ consists in finding the point from which the total weight of the demand points covered (within distance $R$ ) is maximal. This corresponds to the problem discussed by Drezner (1981) and Mehrez-Stulman (1982), where 
only Euclidean distances are considered and no locational constraints are present. $M Q_{\alpha}$ asks for the point where the distance necessary for covering demand points of total weight at least $\alpha$ is minimal. This problem seems to be new in the location literature. Our aim is to show how to fully construct the functions $C^{*}$ and $Q^{*}$ in an efficient way, in other words how to solve problems $M C_{R}$ and $M Q_{\alpha}$ for all values of $R$ and $\alpha$. We call these problems the parametric maxcovering problem and the parametric minquantile problem.

\section{A general algorithm}

The parametric problem $M Q$ belongs to the class of minquantile optimisation problems introduced by the authors in a recent paper (Carrizosa and Plastria, 1995), where a general solution procedure was described. In the context of location theory, as treated here, this procedure may be further detailed. Following notation will be used. For any $I \subseteq\{1, \ldots, p\}, I \neq \emptyset$, let

$$
\begin{aligned}
z_{I} & =\min _{x \in X} \max _{i \in I} f_{i}(x), \\
M_{I} & =\left\{x \in X \mid \forall i \in I: f_{i}(x) \leq z_{I}\right\} \\
X_{I} & =\left\{x \in X \mid \forall i \in I: f_{i}(x)=z_{I}\right\}
\end{aligned}
$$

and consider the optimisation problem $P_{I}$,

$$
\left(P_{I}\right) \max _{x \in X_{I}} \sum_{i=1}^{p} w_{i} t_{i}(x)
$$

where $t_{i}(x)=\left\{\begin{array}{ll}1 & \text { if } f_{i}(x) \leq z_{I} \\ 0 & \text { otherwise }\end{array}\right.$ for $i=1, \ldots, p$.

If $P_{I}$ is feasible, denote by $x_{I}$ an optimal solution, $\alpha_{I}$ the optimal value and $v_{I}:=\left(x_{I}, z_{I}, \alpha_{I}\right)$. As shown by Carrizosa and Plastria (1995) for any $\alpha$ there exists an optimal solution to $M Q_{\alpha}$ of the form $x_{I}$ with $\operatorname{card}(I) \leq 3$. In this case $\alpha \leq \alpha_{I}$. The algorithm of Carrizosa and Plastria (1995), has the following steps:

\section{Algorithm 1.}

\section{Step 1 : Generate the candidate solutions}

Build the list $\mathcal{L}=\left\{v_{I}: I \subseteq\{1, \ldots, p\}, \operatorname{card}(I) \leq 3, P_{I}\right.$ is feasible $\}$. 


\section{Step 2 : Preprocess list $\mathcal{L}$}

Sort the elements in $\mathcal{L}$ in nondecreasing order of $\alpha_{I}$.

\section{Step 3 : Eliminate dominated solutions}

For each $v^{\prime}=\left(x^{\prime}, z^{\prime}, \alpha^{\prime}\right) \in \mathcal{L}$ in turn from the last to the first : Let $v=(x, z, \alpha)$ be the (now) next element in $\mathcal{L}$ (note that by step 2 $\left.\alpha^{\prime} \leq \alpha\right)$ : If $z^{\prime} \geq z$ delete $v^{\prime}$ from $\mathcal{L}$.

After performing the algorithm above, we have obtained an ordered list $\mathcal{L}^{\prime}:=\left\{v_{m} \mid m=1, \ldots, t\right\}$, with $v_{m}=\left(x_{m}, z_{m}, \alpha_{m}\right)$, and thanks to step 2 we have $\alpha_{1}<\alpha_{2}<\ldots<\alpha_{t}=1$. Setting $\alpha_{0}=0, \alpha_{t+1}=+\infty, Q^{*}\left(\alpha_{0}\right)=0$, $Q^{*}\left(\alpha_{t+1}\right)=+\infty$, we have

\section{Theorem 3.1.}

1. $\left.\forall m \in\{1, \ldots, t\}, \forall \alpha \in] \alpha_{m-1}, \alpha_{m}\right], x_{m}$ is an $\alpha$-minquantile point, and $Q^{*}(\alpha)=z_{m}$.

2. $\forall m \in\{1, \ldots, t\}, \forall R \in\left[Q^{*}\left(\alpha_{m}\right), Q^{*}\left(\alpha_{m+1}\right)\left[, x_{m}\right.\right.$ is an $R$-maxcovering point and $C^{*}(R)=\alpha_{m}$.

\section{Proof.}

This is a direct consequence of theorems 2 and 3 in Carrizosa and Plastria (1995), using the fact that the $f_{i}$ are gauges and hence convex, continuous and have compact level sets.

The first part of this theorem indicates that the list $\mathcal{L}^{\prime}$ gives a complete description of $Q^{*}$, and the second part shows how this also solves the parametric maxcovering problem $M C$. Hence, the information gathered in list $\mathcal{L}^{\prime}$ enables us to simultaneously solve both parametric problems, and clearly shows the link between minquantile and maxcovering optimisation: for any radius $R$ we obtain from $\mathcal{L}^{\prime}$ a value $\alpha, \alpha(R)$, and an $\alpha$-minquantile solution which is also a $R$-maxcovering point.

Remark 3.1. $\alpha_{1}=\max _{1 \leq i \leq p} w_{i}$ and $Q^{*}\left(\alpha_{1}\right)=0$.

Indeed list $\mathcal{L}$ will contain all triples $\left(\alpha_{i}, 0, w_{i}\right)$ for $1 \leq i \leq p$. In step 3 all of these will be deleted except one corresponding to the maximal weight. 
Remark 3.2. Denoting by $C(p)$ the complexity of step 1 in the algorithm above the resolution of the parametric problems $M C$ and $M Q$ has a complexity

$$
0\left(\max \left\{p^{3} \log p, C(p)\right\}\right)
$$

\section{Proof.}

Indeed, $\mathcal{L}$ has cardinality $0\left(p^{3}\right)$, and hence step 2 can be done in $0\left(p^{3} \log p\right)$ time; once $\mathcal{L}$ has been sorted step 3 can be performed in $0\left(p^{3}\right)$ time. This gives a total complexity of $0\left(\max \left\{p^{3} \log p, C(p)\right\}\right)$, as asserted.

In particular, as soon as step 1 can be performed in polynomial time, i.e., $C(p)$ is polynomial in $p$, then both $M C$ and $M Q$ can be solved in polynomial time. As shown in sections 5 and 6 , this will be the case of the most popular instances of distances, namely (inflated) Euclidean and polyhedral distances.

Remark 3.3. Both the radius $R$ and the coverage $\alpha$ may be considered as criteria, the first representing a cost, the second representing quality of service. In such a situation, we face the biobjective problem of minimizing $R$ (the cost) and maximizing at the same time $C(R, \cdot)$ (the coverage) by the choice of the location $x \in X$ and the radius $R$. As suggested in Carrizosa and Plastria (1995) the set $S$ of solutions $\left\{x_{m} \mid 1 \leq m \leq t\right\}$ obtained from list $\mathcal{L}^{\prime}$ is a minimal dominating set, i.e. for any $(x, R)$ there exists some $m(1 \leq m \leq t)$ such that $R \geq z_{m}$ and $C(R, x) \leq C\left(z_{m}, x_{m}\right)=\alpha_{m}$. It follows that this biobjective problem may be reduced to a discrete one, with $0\left(p^{3}\right)$ candidate solutions. Any multicriteria decision making method may thus be applied. E.g. in case a utility function $\varphi(R, C(R, x))$ applies, the problem is solved by inspecting only $S$.

Remark 3.4. The general algorithm remains valid when during step 1 any number of triplets $(x, R, C(R, x))$ are added to list $\mathcal{L}$.

Indeed by the previous remark all of these will be deleted in step 3 . 


\section{Solving the subproblem $P_{I}$}

The general algorithm calls for repeated solution of problems of type $P_{I}$

$$
\left(P_{I}\right) \quad \max _{x \in X_{I}} \sum_{i=1}^{p} w_{i} t_{i}(x)
$$

where $\quad t_{i}(x)=\left\{\begin{array}{ll}1, & \text { if } f_{i}(x) \leq z_{I} \\ 0, & \text { otherwise }\end{array}\right.$, for $i=1, \ldots, p$

The following results show that, whatever the gauges are, each $P_{I}$ can be solved by evaluating a set $C_{I}$ of candidate points, with cardinality $0(p)$. Furthermore, such $C_{I}$ is easily obtained once the minmax problem corresponding to $I$ has been solved.

Lemma 4.1. Let $I \subseteq\{1, \ldots, p\}, I \neq \emptyset$. The set $M_{I}=\{x \in X \mid \forall i \epsilon$ $\left.I: f_{i}(x) \leq z_{I}\right\}$ is a nonempty compact segment. It is a singleton when all gauges $\gamma_{i}$ are round.

\section{Proof.}

First observe that $M_{I}$ is included in the set $\bigcup_{i \in I}\left\{x \in \mathbf{R}^{2} \mid f_{i}(x)=z_{I}\right\}$, which has empty interior. Hence, $M_{I}$ has also empty interior. As the functions $f_{i}$ are translated gauges, it follows that $M_{I}$ is a nonempty compact convex set, thus $M_{I}$ is a segment. It was shown in Pelegrín, Michelot and Plastria (1985) that a minmax location problem with round gauges always has a unique optimal solution, whence the last assertion.

For any segment $Y$, denote by $\operatorname{ext}(Y)$ the set of its extreme points. By convention we assume that the empty set is a segment, with $\operatorname{ext}(\emptyset)=\emptyset$. Recall that $X_{I}=\left\{x \in X \mid \forall i \in I: f_{i}(x)=z_{I}\right\}$.

Lemma 4.2. Let $I \subseteq\{1, \ldots, p\}, I \neq \emptyset$. If $X_{I} \neq M_{I}$, then $X_{I} \subseteq \operatorname{ext}\left(M_{I}\right)$.

\section{Proof.}

The result is evident if $M_{I}$ is a singleton : in that case,

$$
X_{I} \subseteq M_{I}=\operatorname{ext}\left(M_{I}\right)
$$

So we can assume that $M_{I}$ is not a singleton. By lemma $4.1, M_{I}$ is then a nondegenerate segment, the extreme points of which are some $y_{1}, y_{2} \in X$. 
We are going to show that, if $X_{I}$ is not contained in $\operatorname{ext}\left(M_{I}\right)=\left\{y_{1}, y_{2}\right\}$, then $M_{I}=X_{I}$. Hence, suppose that there exists $x \in X_{I} \backslash\left\{y_{1}, y_{2}\right\}$. As $M_{I}$ is a segment and $X_{I} \subseteq M_{I}$, there exists a $\left.\lambda \in\right] 0,1\left[\right.$ such that $x=(1-\lambda) y_{1}+\lambda y_{2}$. For any $i \in I$ we have :

$$
\begin{aligned}
& z_{I}=f_{i}(x)=f_{i}\left((1-\lambda) y_{1}+\lambda y_{2}\right) \quad \text { by definition of } X_{I} \\
& \leq(1-\lambda) f_{i}\left(y_{1}\right)+\lambda f_{i}\left(y_{2}\right) \quad \text { by convexity of } f_{i} \\
& \leq \quad(1-\lambda) z_{I}+\lambda z_{I}=z_{I} \quad \text { by definition of } z_{I} \\
& =\quad z_{I}
\end{aligned}
$$

Thus $f_{i}\left(y_{1}\right)=f_{i}\left(y_{2}\right)=z_{I}$ for all $i \in I$, i.e.: $\operatorname{ext}\left(M_{I}\right) \subseteq X_{I}$. Now, for any $y \in M_{I} \backslash\left\{y_{1}, y_{2}, x\right\}$ it follows that $x$ is contained in the open segment whose extreme points are $y$ and a point in $\operatorname{ext}\left(M_{I}\right), y_{1}$ say. Then there exists $\mu \in] 0,1\left[\right.$ such that $x=(1-\mu) y_{1}+\mu y$ and for any $i \in I$ we have

$$
z_{I}=f_{i}(x) \leq(1-\mu) f_{i}\left(y_{1}\right)+\mu f_{i}(y) \leq(1-\mu) z_{I}+\mu z_{I}=z_{I}
$$

Thus $y \in X_{I}$ for all $y \in M_{I}$, i.e.: $M_{I} \subseteq X_{I}$. As $M_{I} \supset X_{I}$, we would have $M_{I}=X_{I}$.

Theorem 4.1. Let $I \subseteq\{1, \ldots, p\}$ be such that $P_{I}$ is feasible $\left(X_{I} \neq \emptyset\right)$. Then the set

$$
C_{I}=\operatorname{ext}\left(M_{I}\right) \cup \bigcup_{k \notin I} \operatorname{ext}\left(\left\{x \in M_{I} \mid f_{k}(x) \leq z_{I}\right\}\right)
$$

contains an optimal solution to $P_{I}$.

\section{Proof.}

If $X_{I} \subseteq \operatorname{ext}\left(M_{I}\right)$, there is nothing to show. So, we can assume that $X_{I}$ is not included in $\operatorname{ext}\left(M_{I}\right)$, or by lemma 4.2 that $X_{I}=M_{I}$. Let $x \in X_{I}=M_{I}$, $K=\left\{k \notin I \mid f_{k}(x) \leq z_{I}\right\}$, and $Y_{K}=\left\{y \in M_{I}\left|f_{k}(y) \leq z_{I}\right| \forall k \in\right.$ $K\}$. Since $Y_{K}=M_{I} \cap \bigcap_{k \in K^{K}}\left\{y \in \mathbf{R}^{2} \mid f_{k}(y) \leq z_{I}\right\}$ and $x \in Y_{K}$, it follows that $Y_{K}$ is a nonempty closed convex segment, with $\operatorname{ext}\left(Y_{K}\right) \subseteq C_{I}$. Furthermore, $\sum_{j=1}^{p} w_{j} t_{j}(x)=\sum_{j \in I \cup K^{-}} w_{j} \leq \sum_{j=1}^{p} w_{j} t_{j}(y)$ for any $y \in Y_{K}$ and in particular for $y \in \operatorname{ext}\left(Y_{K}\right) \subseteq C_{I}$. Hence,

$$
\max _{x \in X_{I}} \sum_{j=1}^{p} w_{j} t_{j}(x) \leq \max _{y \in C_{I}} \sum_{j=1}^{p} w_{j} t_{j}(y) \leq \max _{x \in M_{I}} \sum_{j=1}^{p} w_{j} t_{j}(x)
$$


as asserted and the result follows from $X_{I}=M_{I}$.

This theorem shows that finding a solution to $P_{I}$ reduces to inspection of at most $2 p$ points on the segment $M_{I}$. This shows that as soon as each $z_{I}$ and $C_{I}$ can be obtained in polynomial time, step 1 in the general algorithm (and hence the whole algorithm) can be performed in polynomial time. In the next two sactions this is shown to be possible in presence of linear constraints for inflated Euclidean norms and also for polyhedral gauges.

\section{The inflated Euclidean-norm case}

Let $X$ be a polyhedron (not necessarily bounded) in $\mathbb{R}^{2}$. Assume that, for all $i, 1 \leq i \leq p, \gamma_{i}=\lambda_{i}\|$.$\| , where \|$.$\| is the Euclidean norm, and \lambda_{i}>0$. Different values of $\lambda_{i}$ might indicate differences in speed due to the use of different transportation modes. For $X=\mathbb{R}^{2}$, algorithms for problem $M C_{R}$ ( $R$ fixed) have been discussed by Drezner (1982), with complexity $0\left(p^{2} \log p\right)$, and by Chazelle and Lee (1986) for the unweighted case, with complexity $0\left(p^{2}\right)$. Tamir (1993) pointed out to us that this latter method may be extended to the weighted case without deterioration of the complexity. However, these techniques cannot be used for sensitivity analysis in $R$, and do not give any information regarding minquantile problems. The following properties of the norm and the polyhedral nature of $X$ enable an explicit construction of the list $\mathcal{L}$ in step 1 of the algorithm:

Property 1 The Euclidean norm is strictly convex. Hence, any minmax problem has a unique solution, i.e.: for any $I, M_{I}$ is a singleton.

Property 2 For any $i, j, 1 \leq i \leq j \leq p$, the mediatrix $\operatorname{med}(i, j)$ between $a_{i}$ and $a_{j}$

$$
\operatorname{med}(i, j)=\left\{x \in \mathbb{R}^{2} \mid \lambda_{i}\left\|x-a_{i}\right\|=\lambda_{j}\left\|x-a_{j}\right\|\right\}
$$

can be easily determined. Indeed, it is well known that, if $\lambda_{i}=\lambda_{j}$, med $(i, j)$ is the straight line orthogonal to the segment connecting $a_{i}$ and $a_{j}$ and passing through its midpoint. On the other hand, if $\lambda_{i} \neq \lambda_{j}$, then $\operatorname{med}(i, j)$ is the circle centered at

$$
\frac{\lambda_{i}^{2}}{\lambda_{i}^{2}-\lambda_{j}^{2}} a_{i}+\frac{-\lambda_{j}^{2}}{\lambda_{i}^{2}-\lambda_{j}^{2}} a_{j}
$$


and with radius

$$
\frac{\lambda_{i} \lambda_{j}}{\lambda_{i}^{2}-\lambda_{j}^{2}}\left\|a_{i}-a_{j}\right\|
$$

(Appolonius circle, also called equicircle by Hearn and Vijay (1982)).

Property 3 Intersections of mediatrices with bd $(X)$, the boundary of $X$, and orthogonal projections of points onto $X$ can be obtained in constant time ( $X$ being considered to be fixed).

Property 4 The optimal solution $Y_{I}$ to the unconstrained problem

$$
\min _{x \in \mathbb{R}^{2}} \max _{i \in I} \lambda_{i}\left\|x-a_{i}\right\|
$$

can be explicitly obtained for any $I, 1 \leq \operatorname{card}(I) \leq 2$. Indeed, if $\operatorname{card}(I)=1, I=\{i\}$, say, $Y_{I}=a_{\imath} ;$ if $\operatorname{card}(I)=2, I=\{i, j\}$, say, it is easily seen that $Y_{I}=\frac{\lambda_{i}}{\lambda_{i}+\lambda_{j}} a_{i}+\frac{\lambda_{1}}{\lambda_{i}+\lambda_{j}} a_{j} \in \operatorname{med}(i, j)$.

Property 5 In order to build the list $\mathcal{L}$ it therefore suffices to study separately the different values 1,2 and 3 for card $(I)$ as follows.

Case $\operatorname{card}(I)=1, I=\{i\}$

If $a_{i} \in X$, then $x_{I}=a_{i}$; otherwise, $x_{I}$ is the orthogonal projection of $a_{i}$ onto $X$, which can be determined, in constant time for fixed $X$.

Case $\operatorname{card}(I)=2, I=\{i, j\}$

If the point $\frac{\lambda_{i}}{\lambda_{i}+\lambda_{j}} a_{i}+\frac{\lambda_{j}}{\lambda_{i}+\lambda_{j}} a_{j}$ (the optimal solution to the unconstrained minmax problem) is in $X$, then it is $x_{I}$. If this is not the case then $x_{l}$ evidently must be a boundary point of $X$. As $P_{I}$ is feasible only if $M_{I} \subseteq \operatorname{med}(i, j)$ (by definition of $X_{I}$ ), we only have to take as candidate points

- the point in $\operatorname{med}(i, j) \cap \mathrm{bd}(X)$, closest to $Y_{I}$ if $\operatorname{med}(i, j)$ is a circle $\left(\lambda_{i} \neq \lambda_{j}\right)$.

- the point of $\operatorname{med}(i, j) \cap \operatorname{bd}(X)$ closest to $\frac{a_{z}+a_{2}}{2}$ when $\operatorname{med}(i, j)$ is a line $\left(\lambda_{i}=\lambda_{j}\right)$.

Case $\operatorname{card}(I)=3, I=\{i, j, k\}$

Any solution to $P_{I}$ must be a common point of $\operatorname{med}(i, j), \operatorname{med}(j, k)$ and med $(i, k)$. For the intersection of these three mediatrices only three cases may arise: 
empty Then $P_{I}$ is infeasible.

singleton If the intersection point is in $X$ then it is $x_{I}$. If it is not $P_{I}$ is infeasible.

pair Now both intersection points are to be checked for inclusion in $X$ and if so included (together with their corresponding distance to $a_{i}$ and covered weight) to list $\mathcal{L}$. Note that in this last case remark 3.4 of section 3 will apply.

Theorem 5.1. When all gauges are inflated Euclidean distances, the parametric problems $M C$ and $M Q$ under linear constraints can be solved in $0\left(p^{4}\right)$ time ( $X$ fixed).

\section{Proof.}

By remark 3.2 of section 3 , we only have to show that step 1 can be performed in $0\left(p^{4}\right)$ time. For any $I, 1 \leq \operatorname{card}(I) \leq 3$, finding the $x_{I}$ (if some exists, and anyway there will be at most 2 ) and the corresponding $z_{I}$ can be performed in constant time ( $X$ fixed), while determining $\alpha_{I}$ (i.e.: finding $\left.v_{I}\right)$ can be done in $0(p)$ time. As we have to process $0\left(p^{3}\right)$ subsets $I$, step 1 can be performed in $0\left(p^{4}\right)$ time

A more efficient method of complexity $O\left(p^{3} \log p\right)$ will be described in a forthcoming paper.

We end this section with an illustration of how the procedure described above works.

\begin{tabular}{||c|c|c|c||}
\hline$i$ & $a_{i}$ & $\omega_{i}$ & $\lambda_{i}$ \\
\hline 1 & $(-10,-10)$ & $3 / 19$ & 1 \\
2 & $(10,-10)$ & $4 / 19$ & 1 \\
3 & $(10,10)$ & $5 / 19$ & 2 \\
4 & $(-10,10)$ & $7 / 19$ & 1 \\
\hline
\end{tabular}

Table 1: Data for the example

Example 5.1. Let $A$ consist of the four vertices of the square $[-10,10] \times$ $[-10,10]$. For $i=1,2,3,4$, let $\omega_{i}$ be the weight associated with $a_{i}$, and $\lambda_{i}$ the inflation factor associated with $\gamma_{i}$, i.e. $\gamma_{i}=\lambda_{i}\|\cdot\|$. These data are given in Table 1 . Let $X=\mathbb{R}^{2}$, so that the problem is unconstrained. We first build the list $\mathcal{L}$ of step 1 . 


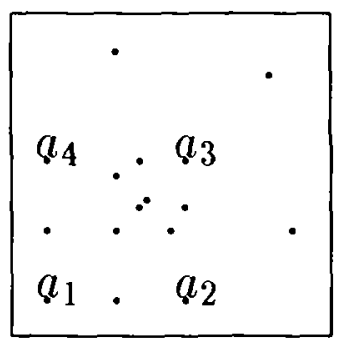

Figure 1: Candidate points

By Property 1, the minmax problems have a unique solution, so there is no need to solve the subproblems $P_{I}$. Moreover, by remark 3.4, the general algorithm remains valid if triplets $(x, R, C(R, x))$ are added to $\mathcal{L}$, so we construct all the triplets $(x, z, \alpha)$ described in Property 5 . The output is given in Table 2.

\begin{tabular}{||c|c|c|c||}
\hline$I$ & $x_{I}$ & $z_{I}$ & $C\left(z_{I}, x_{I}\right)$ \\
\hline$\{1\}$ & $(-10,-10)$ & 0 & $3 / 19$ \\
$\{2\}$ & $(10,-10)$ & 0 & $4 / 19$ \\
$\{3\}$ & $(10,10)$ & 0 & $5 / 19$ \\
$\{4\}$ & $(-10,10)^{*}$ & 0 & $7 / 19$ \\
$\{1,2\}$ & $(0,-10)$ & 10 & $7 / 19$ \\
$\{1,3\}$ & $(3.33,3.33)^{*}$ & 18.86 & $19 / 19$ \\
$\{1,4\}$ & $(-10,0)^{*}$ & 10 & $10 / 19$ \\
$\{2,3\}$ & $(10,3.33)$ & 13.33 & $9 / 19$ \\
$\{2,4\}$ & $(0,0)$ & 14.14 & $14 / 19$ \\
$\{3,4\}$ & $(3.33,10)^{*}$ & 13.33 & $12 / 19$ \\
$\{1,2,3\}$ & $(0,25.49)$ & 36.87 & $19 / 19$ \\
$\{1,2,3\}$ & $(0,7.85)$ & 20.46 & $19 / 19$ \\
$\{1,2,4\}$ & $(0,0)^{*}$ & 14.14 & $14 / 19$ \\
$\{1,3,4\}$ & $(25.49,0)$ & 36.87 & $19 / 19$ \\
$\{1,3,4\}$ & $(7.85,0)$ & 20.46 & $19 / 19$ \\
$\{2,3,4\}$ & $(22.15,22.15)$ & 34.37 & $16 / 19$ \\
$\{2,3,4\}$ & $(4.51,4.51)^{*}$ & 15.52 & $16 / 19$ \\
\hline
\end{tabular}

Table 2: The triplets

In Figure 1 the four demand points (solid dots) and the points $x_{I}$ are plotted. For these points $x_{I}$, the corresponding pairs $\left(z_{I}, C\left(z_{I}, x_{I}\right)\right)$ are 


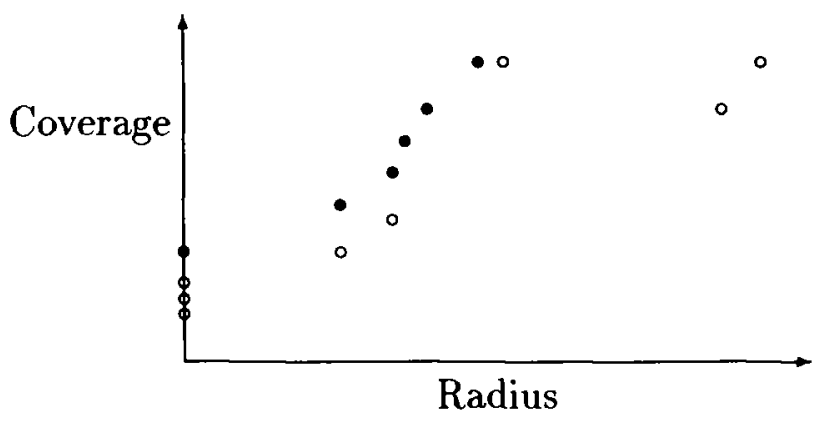

Figure 2: The pairs $\left(z_{I}, C\left(z_{I}, x_{I}\right)\right)$

plotted in Figure 2. Solid circles show the nondominated outcomes (thus yielding elements of the list $\mathcal{L}^{\prime}$ ), whereas empty circles are associated with the triplets $v$ which are deleted in step 3 of the algorithm. This leads to the list $\mathcal{L}^{\prime}$, the elements of which are marked with an asterisk in Table 2, and plotted in Figure 3 (please compare with Figure 1).

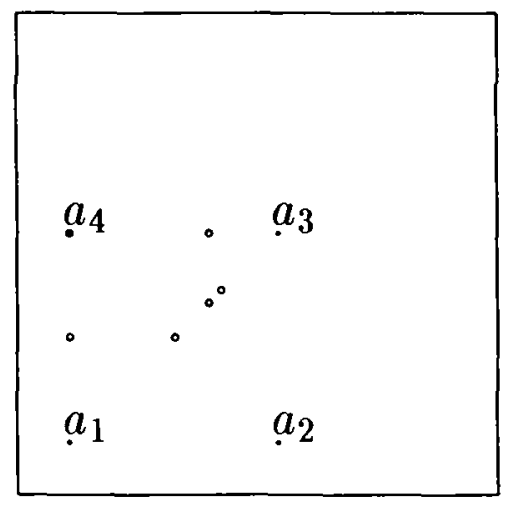

Figure 3: Points in list $\mathcal{L}^{\prime}$

With this information, Theorem 3.1 enables us to give, for each $\alpha$ and $R$ an $\alpha$-minquantile and an $R$-maxcovering point. This is illustrated in Table 3 , where, for the different ranges of values of $\alpha$ and $R$ optimal points (from $\mathcal{L}^{\prime}$ ) are obtained. 


\begin{tabular}{||c|c|c||}
\hline$\alpha$ & $Q^{*}(\alpha)$ & $x^{*}$ \\
\hline$[0,7 / 19]$ & 0 & $(-10,10)$ \\
] $7 / 19,10 / 19]$ & 10 & $(-10,0)$ \\
] $10 / 19,12 / 19]$ & 13.33 & $(3.33,10)$ \\
] $12 / 19,14 / 19]$ & 14.14 & $(0,0)$ \\
] $14 / 19,16 / 19]$ & 15.52 & $(4.51,4.51)$ \\
] $16 / 19,1]$ & 18.86 & $(3.33,3.33)$ \\
\hline
\end{tabular}

\begin{tabular}{||c|c|c||}
\hline$R$ & $C^{*}(R)$ & $x^{*}$ \\
\hline$[0,10[$ & $7 / 19$ & $(-10,10)$ \\
{$[10,13.33[$} & $10 / 19$ & $(-10,0)$ \\
{$[13.33,14.14[$} & $12 / 19$ & $(3.33,10)$ \\
{$[14.14,15.52[$} & $14 / 19$ & $(0,0)$ \\
{$[15.52,18.86[$} & $16 / 19$ & $(4.51,4.51)$ \\
{$[18.16,+\infty[$} & $19 / 19$ & $(3.33,3.33)$ \\
\hline
\end{tabular}

Table 3: Solution of parametric problems

\section{The polyhedral-gauge case}

Suppose that $X$ is a closed polyhedron and every $\gamma_{i}$ is a polyhedral gauge, i.e. a gauge whose unit ball is a polyhedron.

Theorem 6.1. When all gauges are polyhedral, the parametric problems $M C$ and $M Q$ under linear constraints can be solved in $0\left(p^{5}\right)$ time $(X$ fixed).

\section{Proof.}

By remark 3.2 , we only have to show that step 1 in the algorithm can be performed in $0\left(p^{5}\right)$ time. Given $I$, with $1 \leq \operatorname{card}(I) \leq 3$, we first have to solve the problem

$$
\min _{x \in X} \max _{i \in I} f_{i}(x)
$$

Let $E_{i}$ denote the set of extreme points of the dual gauge of $\gamma_{i}$, in other words the normals to the edges of $\gamma_{i}$ 's unit ball. It is well known (see e.g. Durier and Michelot (1985), that $f_{i}(x)=\gamma_{i}\left(x-a_{i}\right)=\max _{e \in E_{i}}\left\langle e, x-a_{i}\right\rangle$, where $\langle\cdot, \cdot\rangle$ denotes usual scalar product. Hence our min-max problem may be written as

$$
\begin{aligned}
& \min t \\
& t \geq\left\langle e, x-a_{i}\right\rangle \quad \forall e \in E_{i}, i \in I \\
& x \in X
\end{aligned}
$$

which is a linear program in $\mathbb{R}^{3}$, solvable in $0(1)$ time, yielding $z_{I}$ and $M_{I}$. For any $k \in\{1, \ldots, p\} \backslash I$, the set $\operatorname{ext}\left(\left\{x \in M_{I} \mid \gamma_{k}(x) \leq z_{I}\right\}\right)$ can be obtained in $0(1)$ time. Hence, $C_{I}$ can be obtained in $0(p)$ time and consists of $0(p)$ points. For any $x \in C_{I}$, the evaluation of $\sum_{i=1}^{p} w_{i} t_{i}(x)$ requires 
$0(p)$ time, thus finding an optimal solution to $P_{I}$ requires a total time of $0\left(p^{2}\right)$. As the number of problems of the form $P_{I}$ to be solved is $0\left(p^{3}\right)$, we conclude that step 1 can be performed in $0\left(p^{5}\right)$ time, as asserted

\section{Further complexity remarks}

The complexity in sections 5 and 6 considered the feasible set $X$ to be fixed. When one considers the description of $X$ as part of the input the complexity of our Euclidean norm algorithm becomes $0\left(p^{2}\left(n+p^{2}\right)\right)$, where $n$ denotes the number of edges of $X$.

In order to state such a 'full input complexity' for the polyhedral gauges case, one has in addition to consider the description of the gauges as part of the input. If one assumes that all gauges used have a unit ball containing at most $0(m)$ vertices, then the overall complexity of our algorithm may be shown to be $0\left(p^{3}\left(n+p m+p^{2} \log m\right)\right)$.

The methodology proposed in this paper may be extended to the location of several facilities. The maximal $k$-covering problem is to determine the location of $k$ facilities, which cover together within a given radius a set of demand points of maximal weight. Similarly the minimal $k$-quantile problem involves locating $k$ facilities minimising the radius needed to cover together a set of demand points of at least a given weight. It is easy to show that optimal locations for both these problems (for any radius or any weight) exist within the candidate solution points constructed in step 1 of the general algorithm. Therefore it will suffice to inspect all sets of cardinality $k$ chosen among these candidate points, leading, for fixed $k$, to a polynomial algorithm for the parametric $k$-covering and $k$-quantile problems in the Euclidean or polyhedral gauges case.

\section{References}

Carrizosa, E. and F. Plastria (1995). On minquantile and maxcovering optimisation. Mathematical Programming, 71, 101-112.

Chazelle, B. and D.T. Lee (1986). On a circle placement problem. Computing, 36, 1-16.

Church, R. and C. ReVelle (1974). Maximal covering location problem. Papers of Regional Science Association, 32, 101-118. 
Drezner, Z. (1981). On a modified one-center model. Management Science, 27, 848-851.

Durier, R. and C. Michelot (1985). Geometrical properties of the Fermat-Weber problem. European Journal of Operational Research, 20, 332-343.

Hearn, D.W. and J. Vijay (1982). Efficient algorithms for the (weighted) minimum circle problem. Operations Research, 30, 777-795.

Mehrez, A. and A. Stulman (1982). The maximal covering location problem with facility placement on the entire plane. Journal of Regional Science, 22, 361365 .

Pelegrín, B., Michelot C. and F. Plastria (1985). On the uniqueness of optimal solutions in continuous location theory. European Journal of Operational Research, 20, 327-331.

Tamir, A. (1993). Private communication. 